\title{
Classification of Brain Tumor using Manta Ray Foraging Optimization-based DeepCNN Classifier
}

\author{
G. Gokulkumari \\ Department of E-Commerce, \\ College of Administrative and Financial Sciences, Saudi Electronic University, \\ An Narjis, Riyadh, Kingdom of Saudi Arabia. \\ gokulkumarig@gmail.com
}

\begin{abstract}
The abnormal cell growth in the brain is the brain tumor. Early diagnosis of brain tumor helps in preventing cancer from advancing to the next level. The major concern in the brain tumor diagnosis is accuracy. In this research, a brain tumor classification method is developed using the Manta Ray Foraging Optimization-based Deep Convolutional Neural Network (MROA-based DeepCNN) algorithm. The preprocessing of Magnetic Resonance (MR) images is done with the Gaussian filter for removing the artifacts in the MR image. The cellular automata and rough set theory are used for segmenting the cancerous region from the preprocessed image. The CNN feature extracted from the segmented output is given as the input to the DeepCNN classifier. The brain tumor is classified into benign, core, edema, and malignant tumor using the DeepCNN classifier, which is trained using the MRFO algorithm. The developed MROA-based DeepCNN method is evaluated using metrics like sensitivity, accuracy, and specificity. While comparing with the existing brain tumor classification methods, the developed MROA-based DeepCNN method obtained a maximum accuracy of 0.9899, maximum sensitivity of 0.8316 , maximum specificity of 0.9899 .
\end{abstract}

Keywords: Brain Tumor, Magnetic Resonance Imaging, Convolutional Neural Network, Gaussian Filter, Brain Tumor Classification.

\section{Introduction}

The brain is the focal part of the nervous system that comprises of non-replaceable and spongy soft tissues. The brain is affected with various diseases, which includes brain tumor. Coordination issues, frequent headaches, difficulty in concentration, memory loss, seizures, and speech changes are the symptoms of a brain tumor. The brain tumor is categorized based on the origin, growth rate, and progression state. According to World Health Organization (WHO), the brain tumor is graded from grade I to grade IV. The features regarding the brain tumor are termed in the allotted grades [4]. MRI is a medical activity used for the diagnosis and analysis of brain tumors. [3]. Due to the presence of features, such as high spatial resolution, high disparity in soft tissues, soft tissue contrast and non-invasive, it is considered as an important imaging modality in the medical field. For brain tumor diagnosis, the MRI is a great source for providing information [9]. The snapshots of the brain are generated by carrying out the tests using computed tomography (CT) or MRI. The nonexistence of radiation in the MRI makes it more effective than a CT scan [10]. The MRI provided augmented knowledge of the contaminated and normal composition of medical research for planning the treatment [11].

The brain MRI classification techniques are classified into two classes, like unsupervised methods and supervised methods [12]. In the supervised classification method, a huge dataset is required with pertinent ground truth. However, the manual gathering of the labeled dataset is a lengthy and difficult task. The supervised classification method used machine learning for the segmentation of brain tumors. One of the commonly used supervised classification methods is SVM. SVM maps the samples vector that are trained into feature space of high dimension and categorized the normal and lesion tissues on the basis of the hyper plane[13]. The unsupervised method adapts to dataset related to diverse imaging protocols, and these methods do not depend on the training dataset [10]. The presence of underprivileged image contrast, mysterious noise, and homogeneity in medical images made the image classification methods not up to grade. During the medical analysis, the accurate classification of medical images is essential, but the classification methods provided complex composition [12]. SVM and random forests are 
the pattern classification methods used for the segmentation of brain tumors. The deep learning methods are employed in image analysis domains for the segmentation of brain tumor in which the object detection, image classification, and semantic segmentation are carried out [14].

The main aim of the research is the development of a brain tumor classification method using MROA-based DeepCNN. Initially, the MR images are preprocessed using a Gaussian filter. Then, the preprocessed MR image is segmented using cellular automata and rough set theory. The segmented output is further used for the feature extraction process in which the CNN features are extracted. Finally, the brain tumor is classified with the DeepCNN classifier. The DeepCNN classifier is trained using the MRFO algorithm for classifying the features into benign, core, edema, and malignant.

- Developed MROA-based DeepCNN: The main contribution of the research is the development of MROA-based DeepCNN for brain tumor classification. The MROA trains the DeepCNN for classifying the brain tumor into a benign, core, edema, and malignant tumor.

The organization of rest of the papers is as follows: section 2 reviews the brain tumor classification methods, section 3 explains the developed MROA-based DeepCNN for brain tumor classification, section 4 discusses the result of developed MROA-based DeepCNN, and section 5 concludes the research.

\section{Motivation}

The literature review of the existing brain tumor classification methods is discussed in this section. The research papers regarding brain tumor classification are considered based on the recent published years, and the challenges faced by the brain tumor classification methods are discussed.

\subsection{Literature Survey}

The review of the literary works is explained below: Pradeep Kumar Mallick et al. [3] developed a brain tumor classification method using Deep Wavelet Autoencoder (DWA)-based Deep Neural Network (DNN). In this method, the property of image decomposition from the wavelet transform was combined with the property of reduction from autoencoder to classify brain tumors. Although this method provided accurate classification results, it had high complexity in computation. Javaria Amin et al. [4] designed CNN for classifying brain tumors. The noise in this research was removed using Partial Differential Diffusion Filter (PDDF). The segmentation was performed using the global thresholding method, and the classification was done with CNN. However, this method failed to classify the brain tumor in large datasets.

Yin, B.et al. [5] modeled an improved version of Whale Optimization Algorithm (IWOA) for the brain tumor classification. The feature selection was performed using logistic mapping and chaos theory. The use of an optimization algorithm in the classification increased the complexity in computation. Siva Raja, P. M., and Rani, A. V. [6] developed a brain tumor classification method using hybrid Deep AutoEncoder with Bayesian Fuzzy Clustering (DAE with BFC) segmentation method. However, this method failed to enhance the classification accuracy.

\subsection{Challenges}

The challenges faced during the classification of brain tumor are as follows:

* The drawback in the DAE with the BFC segmentation approach is the classification accuracy. Thus, the challenge lies in enhancing the accuracy in classification by integrating DNN with other variations of autoencoder for improving the accuracy of the method [3].

- In [6], the major concern is improving the classification accuracy of a large database of medical images. The classification accuracy for a larger database is improved by conjoining with more than one classifier.

- The performance of the multimodal brain tumor classification method depends on the strength of the extracted features. However, the extracted deep learning features increased the time consumption of the execution process. Thus, the challenge lies in enhancing the performance of an automated system by extracting more advanced features [8].

* The focus of the CNN method depends only on the MRI sequences. Thus, the challenge lies in fusing other modalities, like CT and Positron Emission Tomography (PET), for improving the performance of the system [4]. 


\section{Proposed Manta Ray Foraging Optimization-based DeepCNN Classifier for the Classification of Brain Tumor}

This section explains the brain tumor classification method developed using MROA-based DeepCNN. The developed MROA-based DeepCNN has four stages, such as Pre-processing, Tumor segmentation, Feature extraction, and Feature Classification. The first stage is preprocessing, in which the MR image is preprocessed using a Gaussian filter. In the second stage, the tumor is segmented using cellular automata and rough set theory. The third stage is the feature extraction, in which the CNN feature is extracted from the segmented image. The final phase is the feature classification, in which the features are classified using the DeepCNN classifier, which is trained using the MRFO algorithm. Fig. 1 shows the block diagram of the proposed MROA-based DeepCNN for brain tumor classification.

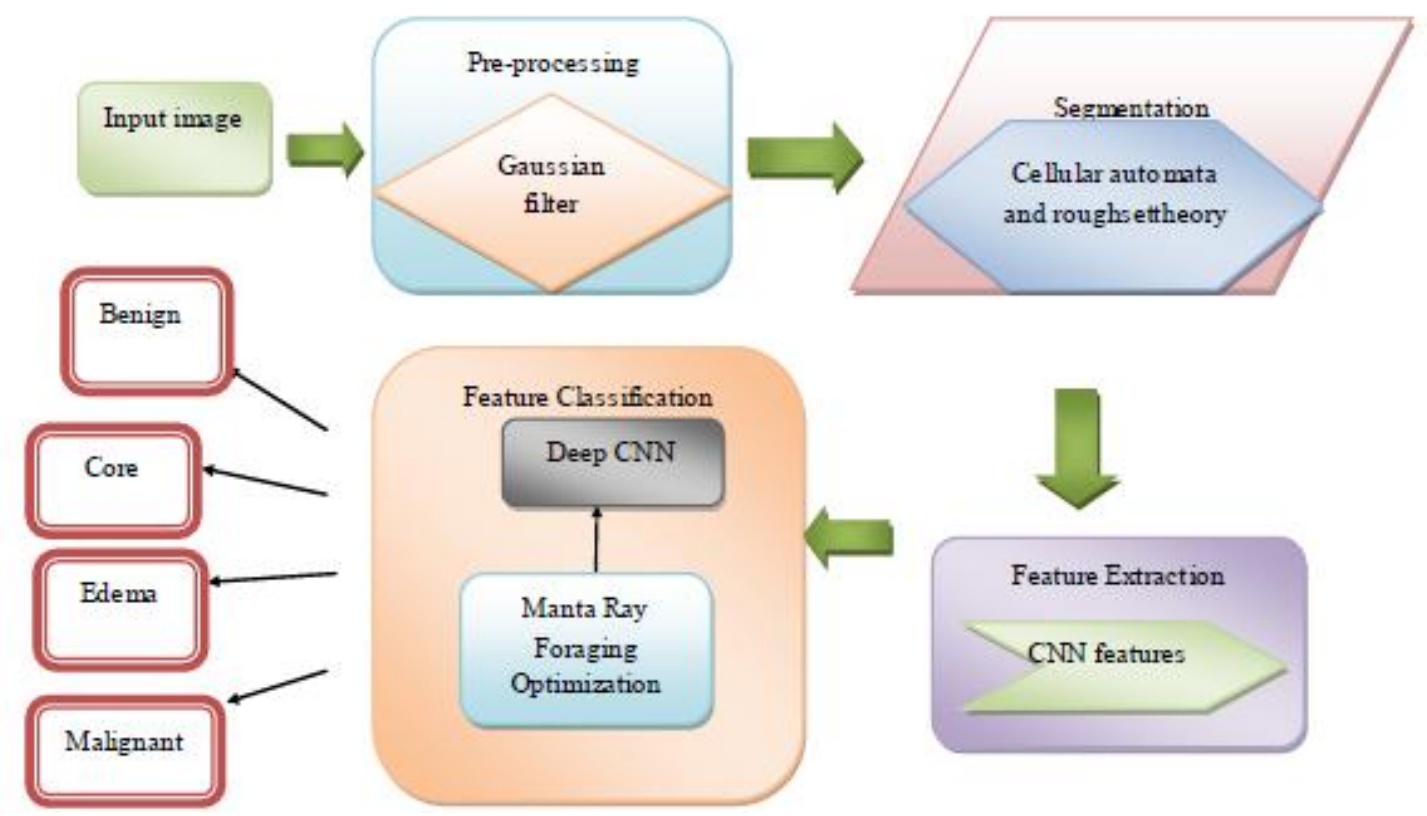

Fig. 1.Block diagram of the proposed MROA-based DeepCNN

The MR image dataset $S$ is considered for brain tumor classification that consists of $b$ MR images. The dataset $S$ is expressed as,

$$
\mathrm{S}=\left\{\mathrm{D}_{1}, \mathrm{D}_{2}, \ldots, \mathrm{D}_{\mathrm{a}}, \ldots, \mathrm{D}_{\mathrm{b}}\right\}
$$

where, the total MR images are represented as, $b$ and the $\mathrm{a}^{\text {th }}$ images in the database are denoted as, $\mathrm{D}_{\mathrm{a}}$.

\subsection{Preprocessing using Gaussian Filter}

Initially, the input MR image $D_{a}$ from the database $S$ is considered for preprocessing. Preprocessing is the process of smoothening the input MR image to make it suitable for the brain tumor classification method. The artifacts and noise in the input MR image are removed with the preprocessing process. In preprocessing, the image enhancement is performed by improving the image contrast. In this research, the Gaussian filter is used for preprocessing the input MR image. In the frequency domain, a smooth transition is provided by the Gaussian filter [1]. The Gaussian filter used the product of 2DGaussian distribution while dealing with images. The 2D Gaussian function is represented as,

$$
\mathrm{B}(\mathrm{U}, \mathrm{V})=\frac{1}{\sqrt{2 \pi \eta^{2}}} \mathrm{e}^{\frac{-\mathrm{U}^{2}+\mathrm{V}^{2}}{2 \eta^{2}}}
$$

where, the filter kernel is denoted as, $W$, the size of filter kernel is represented as, $\mathrm{U}, \mathrm{V}$ and $(-\mathrm{W} \leq \mathrm{U}, \mathrm{V} \leq \mathrm{W})$. The Gaussian distribution's standard deviation is given as, $\eta$. Further, the preprocessed image $L$ obtained from the Gaussian filter is provided as the input to the segmentation process. 


\subsection{Segmentation using Rough set Theory and Cellular Automata}

The preprocessed image $\mathrm{L}$ is further segmented using the automata model and rough set theory [2]. In the segmentation process, the cancerous regions are identified by applying the cellular automata model and rough set theory. In the cellular automata model, the triple function, like $(\mathrm{R}, \mathrm{C}, \chi)$ is used in which $\mathrm{R}$ and $\mathrm{C}$ indicates state and neighbourhood, whereas $\chi$ indicates state transition function. The class label of the pixel is expressed as, Ffor the image segmentation process. For $\mathrm{g}^{\text {th }}$ pixel, the neighbourhood $\mathrm{C}$ is expressed as,

$$
\mathrm{C}=\mathrm{X}(\mathrm{g} ; \mathrm{w}) \cap \mathrm{G}^{2}
$$

From the above expression, the pixel $\mathrm{g}$ for the circle having radius $\mathrm{w} \geq 1$ is denoted as, $\mathrm{X}(\mathrm{g} ; \mathrm{w})$. At the time $\mathrm{h}$, the cell value is considered by the state transition function $\chi$ that referred to the state of the cell at a time $\mathrm{h}+1$. For classifying the non-accurate data that are not characterized under lower boundary or upper boundary, the rough set theory is used. In the rough set theory and cellular automata model, the segments obtained are denoted as,

$$
A=\left\{A_{1}, A_{2}, \ldots, A_{c}, \ldots, A_{e}\right\}
$$

where, the segments obtained in the segmentation approach is denoted as, A .

\subsection{Feature Extraction using CNN}

The segmentation output $\mathrm{A}$ is provided as the input to CNN for obtaining the CNN features. The architecture of CNN consists of convolutional (conv) layer, POOL layer, and Fully Connected (FC) layer. In conv layer, the CNN features are obtained from the segmented image. The relation in between the value of pixel and image features is preserved in conv layer such that the CNN features improved the classification accuracy by enabling the pixel-wise operation. The CNN feature has the dimension of $[1 \times 256]$, which is represented as, $\lambda$.

\subsection{Feature Classification using Deep CNN}

After the extraction of CNN features, the features obtained are classified using the Deep CNN. The Deep CNN is trained using the MROA [15]. The foraging behavior of the manta ray is considered for solving the optimization problems in the MRFO algorithm. The foraging behaviour includes chain foraging, somersault foraging, and cyclone foraging. In the chain foraging operation, an orderly line is formed by the manta ray by lining up from one behind another. A long foraging chain is formed by manta rays during cyclone foraging and they swim towards the food source in a spiral location. In somersault foraging, a series of backwards somersaults are performed by the manta ray after finding the food source, and they circle the food source to draw it towards them. The advantage of MRFO is the low computational cost for solving global optimization problems. Thus, the MRFO algorithm is used for training the Deep CNN. The architecture of Deep CNN is discussed below,

\subsubsection{Architecture of DeepCNN}

The architecture of DeepCNN has three layers, like conv layer, POOL layer, and FC layer. The feature maps are sampled in conv layer, whereas the classification is performed in the FC layer. The input to the DeepCNN is $\lambda$, which is the feature extracted from the segmented image. Fig. 2 explains the architecture of Deep CNN.

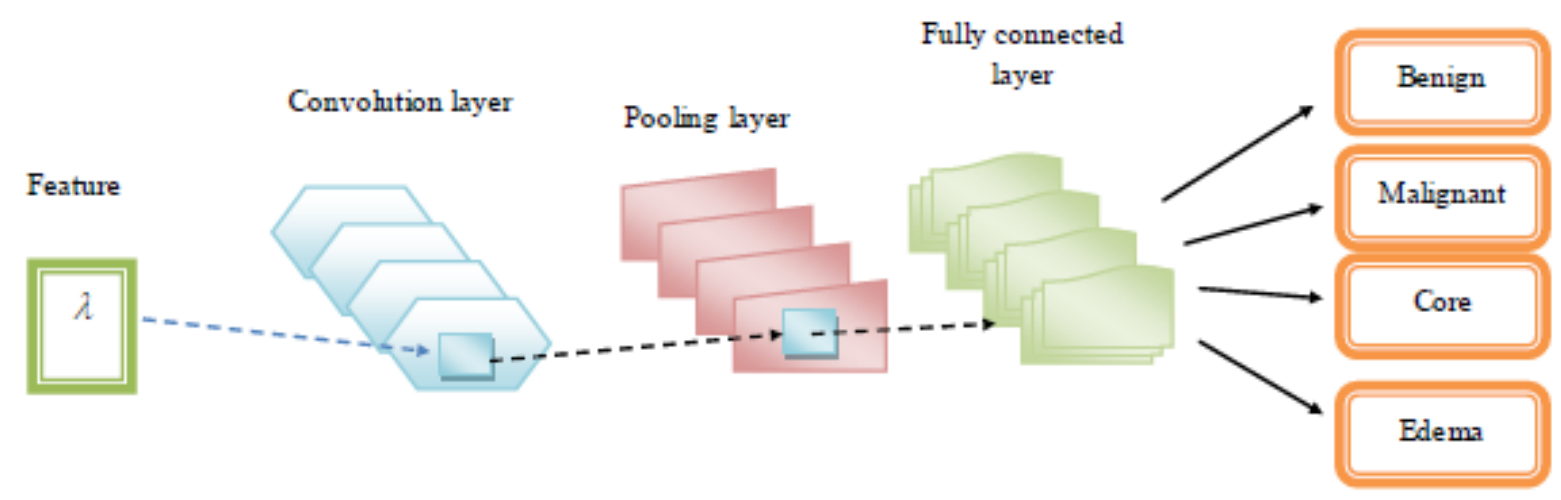

Fig.2. Architecture of Deep CNN 
Conv layers: The conv layer is the first layer in which the feature map is composed by convoluting the inputs with the trainable weights and linking the neurons with the trainable weights. The conv layer output is represented as,

$$
\left(\mathrm{k}_{\mathrm{u}}^{\mathrm{v}}\right)_{\mathrm{x}, \mathrm{y}}=\left(\mathrm{H}_{\mathrm{u}}^{\mathrm{v}}\right)_{\mathrm{x}, \mathrm{y}}+\sum_{\mathrm{f}=1}^{\mathrm{s}_{1}^{\mathrm{f}-1}} \sum_{\gamma=-\mathrm{s}_{1}^{\mathrm{v}}}^{\mathrm{s}_{1}^{\mathrm{p}}} \sum_{\mathrm{l}=-\mathrm{s}_{2}^{\mathrm{p}}}^{\mathrm{s}_{2}^{\mathrm{p}}}\left(\xi_{\mathrm{u}, \mathrm{f}}^{\mathrm{p}}\right){ }_{1, \gamma} *\left(\mathrm{k}_{\mathrm{f}}^{\mathrm{p}-1}\right)_{\mathrm{x}+\gamma, \mathrm{y}+1}
$$

where, $v^{\text {th }}$ conv layer centered around $(\mathrm{x}, \mathrm{y})$ is denoted as, $\left(\mathrm{k}_{\mathrm{u}}^{\mathrm{v}}\right)_{\mathrm{x}, \mathrm{y}}$, conv operator is specified by the term, *. The input to the succeeding $\mathrm{q}^{\text {th }}$ layer is the output from the previous $(\mathrm{p}-1)^{\text {th }}$ layer. In $\mathrm{p}^{\text {th }}$ conv layer, the weight and bias of conv layer is represented as, $\left(\xi_{u, f}^{p}\right)$ and $H_{u}^{v}$. The feature map from the conv filter output is denoted by the term $\mathrm{f}, \gamma$, and 1 . In the ReLU layer, the $\mathrm{q}^{\text {th }}$ layer output forms the activation function of $(q-1)^{\text {th }}$ layer. The optimal region is selected efficiently using the ReLU layer as it provided high speedup.

POOL layers and Fully connected layers: The POOL is a non-parametric layer with no bias and weight. The input to FC is the output derived from the POOL layer. The FC layer output is represented as,

$$
\sigma_{\mathrm{u}}^{\mathrm{v}}=\delta\left(\mathrm{k}_{\mathrm{u}}^{\mathrm{v}}\right)
$$

Consequently, the term $\left(\mathrm{k}_{\mathrm{u}}^{\mathrm{v}}\right)_{\mathrm{x}, \mathrm{y}}$ is represented as,

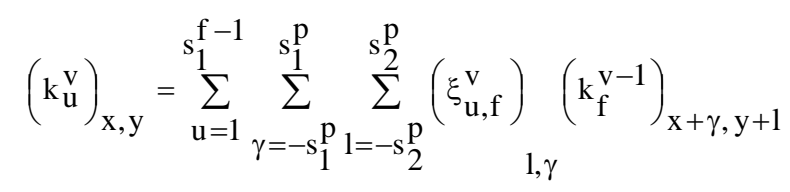

where, the Deep CNN classifier's output is denoted as, $\sigma_{u}^{v}$.Thus, the features are classified into benigntumor, coretumor, edematumor, and malignant tumor using DeepCNN classifier.

\subsubsection{Training of DeepCNN using developed MRFO-based DeepCNN}

The optimal weights derived from MRFO algorithm is used for training Deep CNN for the classification of brain tumor. The algorithmic steps of MRFO-based DeepCNN are as follows,

\section{Step 1: Initialization}

Initially, the manta ray size is assumed as, $\mathrm{J}$ and individual manta ray is represented as,

$$
\mathrm{E}_{\mathrm{m}}^{\tau}=\mathrm{E}_{\mathrm{m}}+\operatorname{rand}(\mathrm{P}-\mathrm{Q})
$$

where, $\mathrm{m}=1, \ldots, \mathrm{J}$ and $\mathrm{t}=1$. The lower and upper boundary is represented as, $\mathrm{P}$ and $\mathrm{Q}$.

\section{Step 2: Determination of Fitness function}

The fitness function is the distance of the source from the manta ray position. The fitness function is calculated by the following equation as,

$$
\operatorname{MSE}=\frac{1}{\mathrm{z}}\left[\sum_{0=1}^{\mathrm{Z}} \mathrm{Q}_{\mathrm{t}} \arg \text { et }-\mathrm{M}\right]
$$

where, estimated output from Deep $\mathrm{CNN}$ and targeted output is represented as, $\mathrm{M}$ and $\mathrm{Q}_{\mathrm{t}}$ arg et, $\mathrm{z}$ is the number of training samples.

\section{Step 3: Chain foraging}

In Chain foraging, the manta ray move towards the food source after determining the position of food source. The food source with a high concentration of plankton is considered as the best position. During chain foraging, the food source and first individual is followed by another manta ray. The manta rays line up from head-to-tail to form a foraging chain. The chain foraging process is expressed as, 


$$
E_{m}^{t+1}=\left\{\begin{array}{l}
E_{m}^{t}+d\left(E_{\text {best }}^{t}-E_{m}^{t}\right)+v\left(E_{\text {best }}^{t}-E_{p}^{t}\right) m=1 \\
E_{m}^{t}+d\left(E_{m-1}^{t}-E_{m}^{t}\right)+v\left(E_{\text {best }}^{t}-E_{m}^{t}\right) m=2, \ldots, J
\end{array}\right.
$$

where, high plankton concentration and weight coefficient is denoted as, $\mathrm{E}_{\text {best }}^{\mathrm{t}}$ and $\mathrm{v}$, respectively, the position of $\mathrm{m}^{\text {th }}$ individual at time $\mathrm{t}$ is given as, $\mathrm{E}_{\mathrm{m}}^{\mathrm{t}}$. On the basis of $(\mathrm{m}-1)^{\text {th }}$ individual's current location and food location $\mathrm{E}_{\text {best }}^{\mathrm{t}}$, the location of $\mathrm{m}^{\text {th }}$ individual is updated.

\section{Step 4: Cyclone foraging}

The manta rays swim towards the food source in spiral motion in deep water. Every individual manta ray follows the manta ray in the front while moving towards the food location. The cyclone foraging process is expressed mathematically as.

$$
E_{m}^{t+1}=\left\{\begin{array}{l}
E_{\text {best }}+d\left(E_{\text {best }}^{t}-E_{m}^{t}\right)+\partial\left(E_{\text {best }}^{t}-E_{m}^{t}\right) m=1 \\
E_{\text {best }}+d\left(E_{\text {best }}^{t}-E_{m}^{t}\right)+\partial\left(E_{\text {best }}^{t}-E_{m}^{t}\right) m=2, \ldots, J
\end{array}\right.
$$

where, $\partial=2 \mathrm{e}^{\mathrm{d}_{1} \frac{\mathrm{K}-\mathrm{t}+1}{\mathrm{~K}}} \cdot \sin \left(2 \pi \mathrm{d}_{1}\right)$, the maximum number of iteration is denoted as, $\mathrm{K}$, the random number between the interval $[0,1]$ is represented as, $d_{1}$. The weight coefficient is given as, $\partial$.By considering the food as the reference location, the individuals perform the search randomly thus, exploiting the region containing the best solution. Then, the individual manta ray is forced to find new location of food source by allocating random position for the individuals in the search space. The cyclone foraging helps in enabling extensive global search. The mathematical expression for cyclone foraging is given as,

$$
E_{m}^{t+1}=\left\{\begin{array}{c}
E_{\text {rand }}=Q+d \cdot(P-Q) \\
E_{\text {rand }}+d\left(E_{\text {rand }}^{t}-E_{m}^{t}\right)+v\left(E_{\text {rand }}^{t}-E_{m}^{t}\right) m=1 \\
E_{\text {rand }}+d\left(E_{\text {rand }}^{t}-E_{m}^{t}\right)+v\left(E_{\text {rand }}^{t}-E_{m}^{t}\right)
\end{array}\right.
$$

where, the lower limit and upper limit in $\mathrm{x}^{\text {th }}$ dimension is represented by the term, $\mathrm{P}$ and $Q$. $\mathrm{E}_{\text {rand }}$ is the position randomly determined in the search space.

\section{Step 5: Somersault foraging}

The location of the food source is assumed as a pivot in somersault foraging behaviour. The individual manta ray swims from side to side around the pivot, thus somersaulting to the new location. The mathematical expression in the somersault foraging cycle is denoted as,

$$
\mathrm{E}_{\mathrm{m}}^{\mathrm{t}+1}=\mathrm{E}_{\mathrm{m}}^{\mathrm{t}}+\mathrm{T}\left(\mathrm{d}_{2} \cdot \mathrm{E}_{\text {best }}-\mathrm{d}_{3} \cdot \mathrm{E}_{\mathrm{m}}^{\mathrm{t}}\right), \mathrm{m}=1, \ldots, \mathrm{J}
$$

where, $\mathrm{T}$ is the somersault factor, which is equal to 2 . The individual manta ray is free to travel in the new search domain, which is located between the current location and the best position of the food source. The individual manta ray moves towards the optimal solution in the search space gradually. The range of somersault foraging reduces as the number of iterations increases.

\section{Step 6: Termination}

The best position is determined and updated until the stopping criterion is met. The obtained best position is used for training the DeepCNN for the classification of brain tumors.

\section{Results and Discussion}

The proposed MROA-based DeepCNN method is analyzed using the sim BRATS and BRATS dataset for the metrics by varying the percentage of training data. 


\subsection{Experimental Setup}

The MROA-based DeepCNN method is implemented in the Matlab tool in a PC having Windows 10 OS. The datasets used for the experimentation of MROA-based DeepCNN method are BRATS and sim BRATS dataset taken from BRATS 2014 [7]. From the image of patients, the modalities, such as T1C, T1, FLAIR, and T2 are accumulated.

\subsection{Evaluation Metrics}

The performance metrics used for the evaluation in the proposed MROA-based DeepCNN method are sensitivity, accuracy, and specificity.

\subsubsection{Sensitivity}

The sensitivity is the ratio of true positive to the sum of a true positive and false negative. The below expression evaluates the sensitivity as,

$$
\text { Sensitivit } y=\frac{T^{p}}{F^{n}+T^{p}}
$$

\subsubsection{Accuracy}

The accuracy is the degree of closeness between the estimated and original value. The accuracy is determined as,

$$
\text { Accuracy }=\frac{T^{n}+T^{p}}{F^{p}+F^{n}+T^{p}+T^{n}}
$$

\subsubsection{Specificity}

The specificity is the ratio of true negative to the sum of false-positive and true negative. The specificity is expressed as,

$$
\text { Specificity }=\frac{\mathrm{T}^{\mathrm{n}}}{\mathrm{T}^{\mathrm{n}}+\mathrm{F}^{\mathrm{p}}}
$$

where, $T^{P}, F^{P}, T^{n}$ and $F^{n}$ represented the true positive, false positive, true negative, and false negative, respectively.

\subsection{Experimental Results}

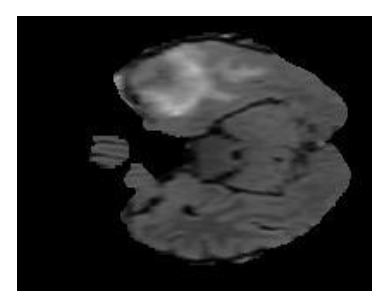

a)

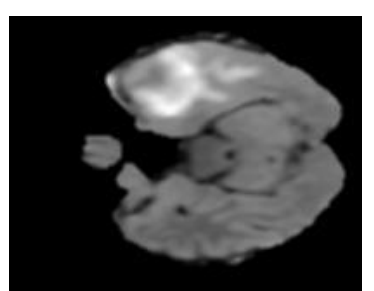

b)

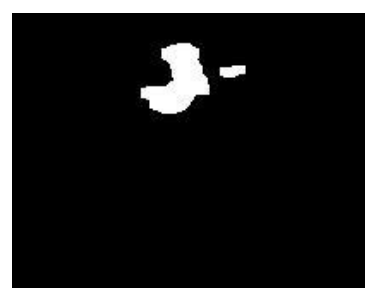

c)

Fig.3. Experimental results a) Original Image, b) Preprocessed Image and c) Segmented output using BRATS dataset

Fig. 3 depicts the experimental results obtained in the developed MROA-based DeepCNN method using the BRATS dataset. Fig. 3 a) shows the Original Image from the BRATS dataset, Fig. $3 \mathrm{~b}$ ) illustrates the preprocessed MR image, Fig. 3 c) explains the segmented output obtained using the CNN features.

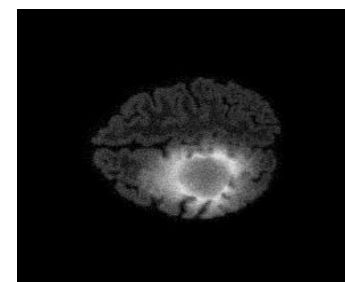

a)

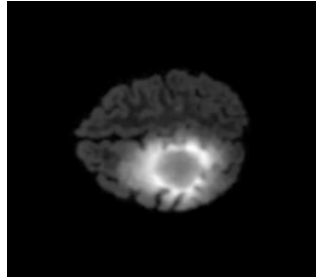

b)

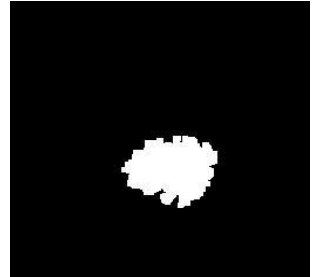

c)

Fig.4. Experimental results a) Original Image, b) Preprocessed Image and c) Segmented output using SimBRATS dataset 
Fig. 4 illustrates the experimental results obtained in the developed MROA-based DeepCNN method using Sim BRATS dataset. Fig. 4 a) depicts the Original Image from the BRATS dataset, Fig. 4 b) explains the preprocessed MR image, Fig. 4 c) shows the segmented output obtained through the cellular automata model and rough set theory.

\subsection{Comparative Analysis}

The developed MROA-based DeepCNN is compared with the existing CNN [4], DWA-based DNN [3], IWOA [5], Hybrid DAE with BFC segmentation methods [6] using the metrics for varying training data.

\subsubsection{Analysis using BRATS dataset}

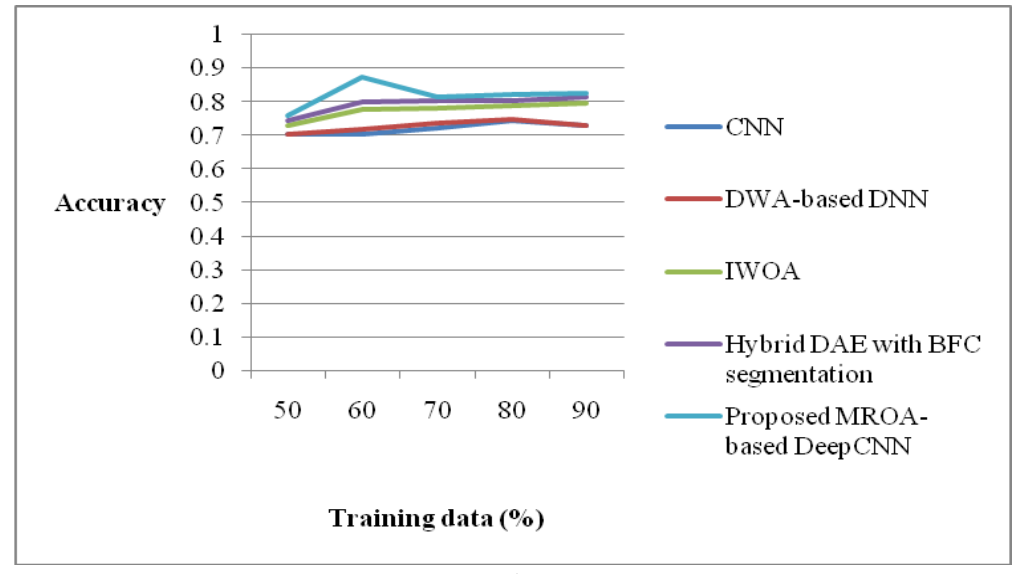

a)

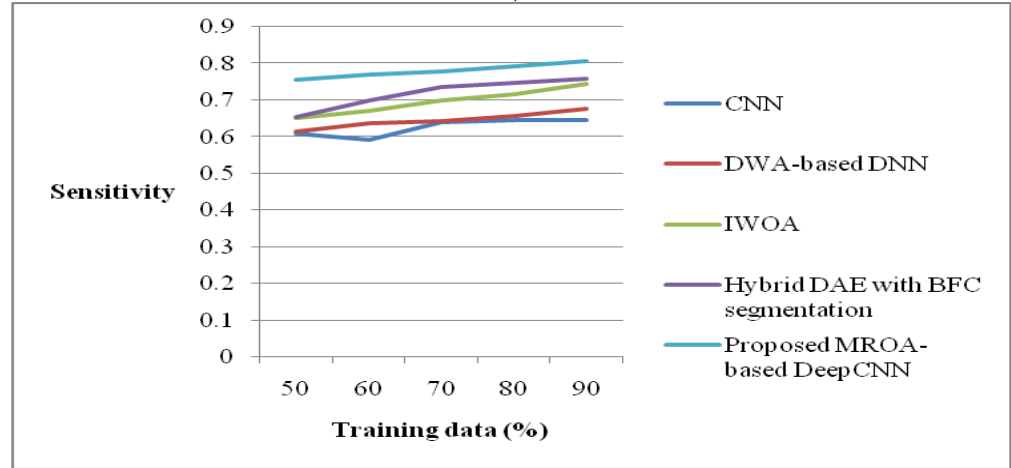

b)

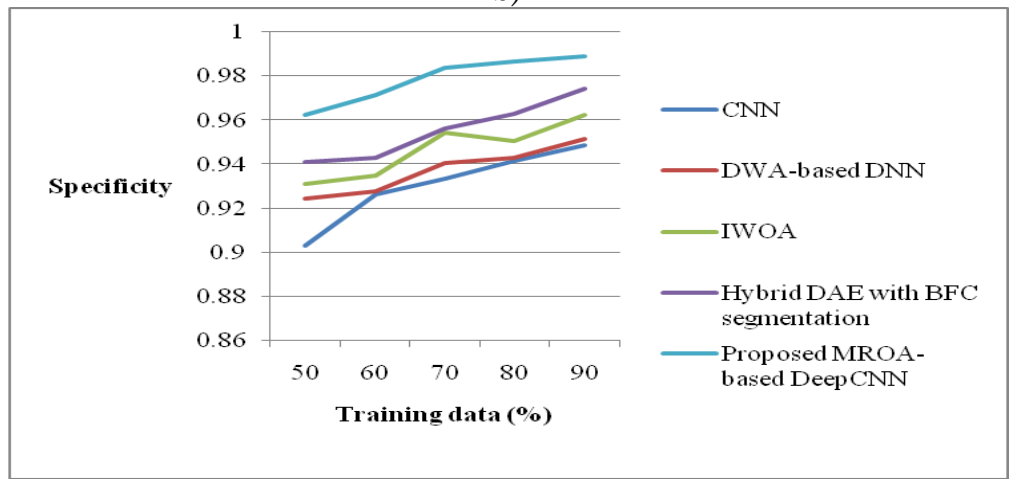

c)

Fig. 5. Analysis of BRATS dataset using a) accuracy, b) sensitivity, and c) specificity

Fig. 5 illustrates the analysis of the BRATS dataset using the metrics for varying training data. Fig. 5 a) depicts the accuracy analysis of brain tumor classification methods. For training data of $50 \%$, the accuracy obtained by the CNN, DWA-based DNN, IWOA, Hybrid DAE with BFC segmentation, and developed MROA-based DeepCNN is 0.7029, 0.7049, 0.7316, 0.7433, and 0.7591, respectively. The performance improvement obtained by the developed MROA-based DeepCNN on comparing with the existing CNN, DWA-based DNN, IWOA, Hybrid DAE with BFC segmentation methods are 7.40\%, 7.14\%, $3.62 \%$, and $2.08 \%$, respectively. Fig. 5 b) shows the sensitivity analysis of brain tumor classification methods. The sensitivity of the CNN, DWA-based DNN, IWOA, Hybrid DAE with BFC segmentation, 
and developed MROA-based DeepCNN for the training data of $80 \%$ is $0.6446,0.6568,0.7146,0.749$, and 0.7917, respectively. On comparing with the CNN, DWA-based DNN, IWOA, Hybrid DAE with BFC segmentation methods, the developed MROA-based DeepCNN obtained a percentage improvement of $18.58 \%, 17.04 \%, 9.74 \%$, and 5.39\%, respectively. Fig. 5 c) depicts the brain tumor classification methods using specificity. The existing CNN, DWA-based DNN, IWOA, Hybrid DAE with BFC segmentation and developed MROA-based DeepCNN obtained a specificity of 0.9337, 0.9403, 0.9543, 0.9566 and 0.9838, respectively for the training data of $70 \%$. The developed MROA-based DeepCNN method obtained a percentage improvement of $5.09 \%, 4.42 \%, 3 \%$, and $2.76 \%$, respectively.

\subsubsection{Analysis using SimBRATS Dataset}

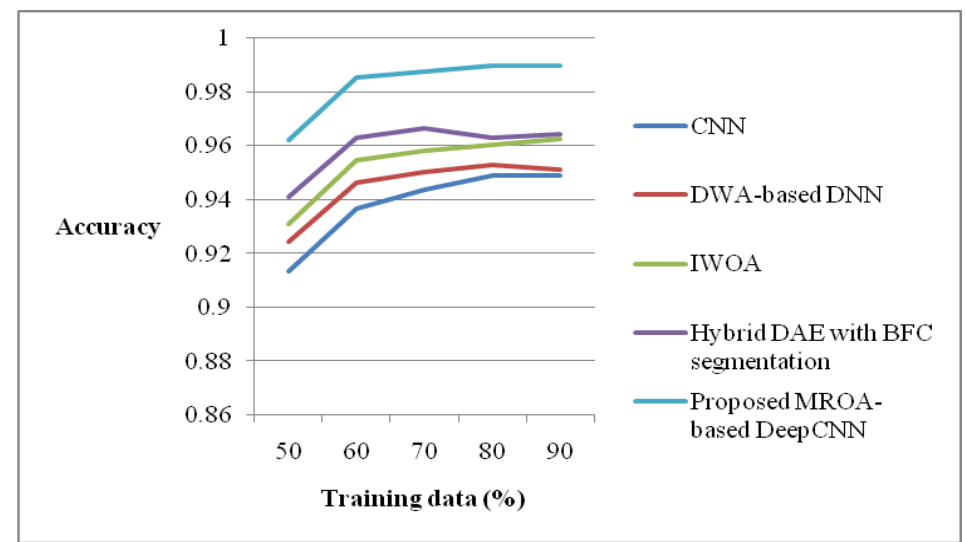

a)

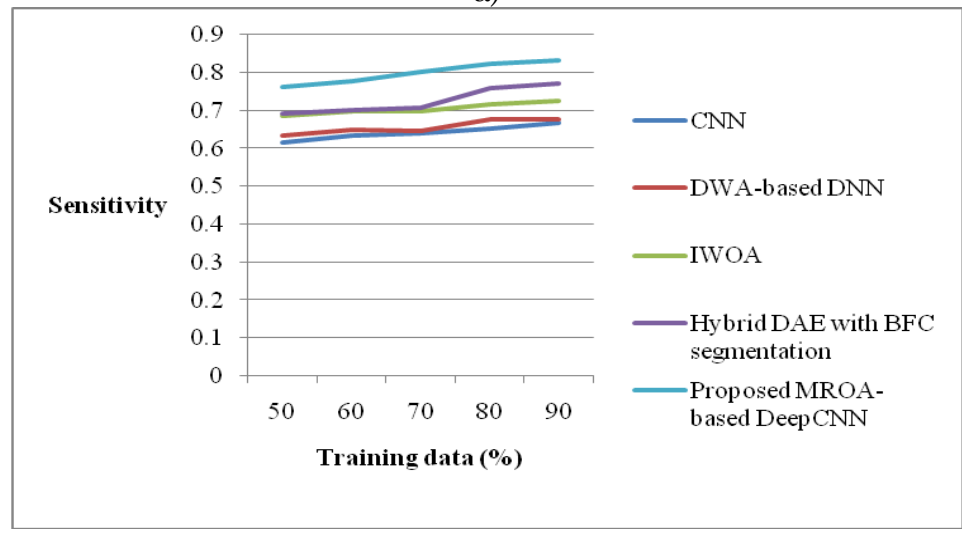

b)

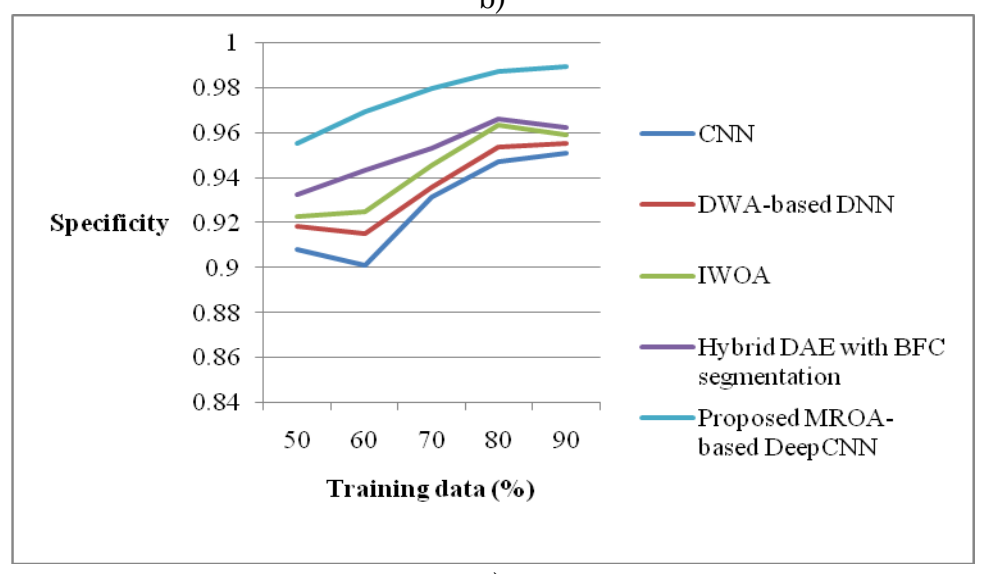

c)

Fig. 6. Analysis of SimBRATS dataset using a) accuracy, b) sensitivity, and c) specificity

Fig. 6 shows the analysis of the SimBRATS dataset using the metrics for varying training data. Fig. 6 a) depicts the accuracy analysis of brain tumor classification methods. The accuracy of the CNN, DWAbased DNN, IWOA, Hybrid DAE with BFC segmentation, and developed MROA-based DeepCNN for the training data of $60 \%$ is $0.9367,0.9464,0.9549,0.9632$, and 0.9855 , respectively. Compared with the CNN, DWA-based DNN, IWOA, Hybrid DAE with BFC segmentation methods, the developed MROAbased DeepCNN obtained a percentage improvement of $4.95 \%, 3.97 \%, 3.11 \%$, and $2.26 \%$, respectively. 
Fig. $6 \mathrm{~b}$ ) describes the sensitivity analysis of brain tumor classification methods. For training data of $50 \%$, the sensitivity obtained by the CNN, DWA-based DNN, IWOA, Hybrid DAE with BFC segmentation, and developed MROA-based DeepCNN is 0.6171, 0.6335, 0.6872, 0.6918, and 0.7614, respectively. The performance improvement obtained by the developed MROA-based DeepCNN on comparing with the existing CNN, DWA-based DNN, IWOA, Hybrid DAE with BFC segmentation methods are $18.95 \%, 16.80 \%, 9.75 \%$ and $9.14 \%$, respectively. Fig. 6 c) depicts the brain tumor classification methods using specificity. When the training data is $70 \%$, the existing CNN, DWA-based DNN, IWOA, Hybrid DAE with BFC segmentation and developed MROA-based DeepCNN method obtained a specificity of $0.9316,0.9358,0.9459,0.9534$ and 0.9803 , respectively. The developed MROAbased DeepCNN method obtained a percentage improvement of $4.97 \%, 4.54 \%, 3.515 \%$, and $2.74 \%$, respectively.

\subsection{Comparative Discussion}

Table 1 depicts the Comparative discussion of brain tumor classification methods using the BRATS dataset for training data of 90\%. The developed MROA-based DeepCNN method obtained a maximum accuracy of 0.8272 , whereas the CNN, DWA-based DNN, IWOA, Hybrid DAE with BFC segmentation methods obtained an accuracy of $0.729,0.7328,0.7982$, and 0.8164 , respectively. When compared with the existing CNN, DWA-based DNN, IWOA, Hybrid DAE with BFC segmentation methods with the sensitivity of $0.646,0.6767,0.7431,0.7595$, the developed MROA-based DeepCNN method obtained a maximum sensitivity of 0.8054 . The specificity obtained by the developed MROA-based DeepCNN method is 0.9792, which is maximum compared when compared to CNN, DWA-based DNN, IWOA, Hybrid DAE with BFC segmentation methods with the specificity of 0.9489, 0.9515, 0.9626, and 0.9743, respectively.

Table 2 shows the Comparative discussion of brain tumor classification methods using the SimBRATS dataset for training data of $90 \%$. When compared with the existing CNN, DWA-based DNN, IWOA, Hybrid DAE with BFC segmentation methods with the accuracy of $0.9489,0.9515,0.9626$, and 0.9643 , the developed MROA-based DeepCNN method obtained a maximum accuracy of 0.9899. The sensitivity obtained by the developed MROA-based DeepCNN method is 0.8316, which is maximum compared when compared to CNN, DWA-based DNN, IWOA, Hybrid DAE with BFC segmentation methods with the sensitivity of $0.6665,0.6771,0.725$, and 0.7715 , respectively. The developed MROAbased DeepCNN method obtained a maximum specificity of 0.9899, whereas the CNN, DWA-based DNN, IWOA, Hybrid DAE with BFC segmentation methods obtained a specificity of $0.9513,0.9553,0.9592$, and 0.9621 , respectively

Table 1. Comparative discussion of brain tumor classification methods using BRATS dataset

\begin{tabular}{lccccc}
\hline \multicolumn{1}{c}{ Metric } & CNN & DWA-based DNN & IWOA & $\begin{array}{l}\text { Hybrid DAE with BFC } \\
\text { segmentation }\end{array}$ & $\begin{array}{c}\text { Proposed MROA- } \\
\text { based DeepCNN }\end{array}$ \\
\hline Accuracy & 0.7290 & 0.7328 & 0.7982 & 0.8164 & $\mathbf{0 . 8 2 7 2}$ \\
Sensitivity & 0.6460 & 0.6767 & 0.7431 & 0.7595 & $\mathbf{0 . 8 0 5 4}$ \\
Specificity & 0.9489 & 0.9515 & 0.9626 & 0.9743 & $\mathbf{0 . 9 8 9 2}$ \\
\hline
\end{tabular}

Table 2. Comparative discussion of brain tumor classification methods using SimBRATS dataset

\begin{tabular}{lccccc}
\hline \multicolumn{1}{c}{ Metric } & CNN & DWA-based DNN & IWOA & $\begin{array}{c}\text { Hybrid DAE with } \\
\text { BFC segmentation }\end{array}$ & $\begin{array}{c}\text { Proposed MROA- } \\
\text { based DeepCNN }\end{array}$ \\
\hline Accuracy & 0.9489 & 0.9515 & 0.9626 & 0.9643 & $\mathbf{0 . 9 8 9 9}$ \\
Sensitivity & 0.6665 & 0.6771 & 0.725 & 0.7715 & $\mathbf{0 . 8 3 1 6}$ \\
Specificity & 0.9513 & 0.9553 & 0.9592 & 0.9621 & $\mathbf{0 . 9 8 9 9}$ \\
\hline
\end{tabular}

\section{Conclusion}

In this research, an MROA-based DeepCNN classifier is developed for the classification of brain tumors. Initially, the preprocessing is performed in the MR images using a Gaussian filter. After preprocessing, the MR image is segmented with the help of cellular automata and rough set theory. Then, the feature, such as CNN feature is extracted from the segmented MR image for further processing. Finally, the features are classified using the DeepCNN classifier. The input to the DeepCNN classifier is CNN features, which is trained with the MRFO algorithm. Thus, the MR images are classified into benign, core, edema and malignant tumor, which is used for the accurate diagnosis of brain tumor. The performance of the developed MROA-based DeepCNN method is evaluated using the metrics such as sensitivity, accuracy and specificity using the SimBRATS dataset and the BRATS dataset. The developed 
MROA-based DeepCNN method obtained a maximum accuracy of 0.9899 , a maximum sensitivity of 0.8316, maximum specificity of 0.9899 , respectively, while comparing with the existing brain tumor classification methods. The developed MROA-based DeepCNN method can be further enhanced by including a more advanced optimization algorithm for training the classifier for accurate brain tumor classification.

\section{Compliance with Ethical Standards}

Conflicts of interest: Authors declared that they have no conflict of interest.

Human participants: The conducted research follows the ethical standards and the authors ensured that they have not conducted any studies with human participants or animals.

\section{References}

[1] Hwang, J.J. and Rhee, K.H., "Gaussian filtering detection based on features of residuals in image forensics," In IEEE RIVF International Conference on Computing \& Communication Technologies, Research, Innovation, and Vision for the Future (RIVF), pp. 153-157, 2016.

[2] D. Ramamurthy and P.K. Mahesh, "Brain Tumor Segmentation based on Rough Set Theory for MR Images with Cellular Automata Approach", January 2019.

[3] Pradeep Kumar Mallick, Seuc Ho Ryu, Sandeep Kumar Satapathy, Shruti Mishra, Nhu Gia Nguyen, and Prayag Tiwari, "Brain MRI Image Classification for Cancer Detection using Deep Wavelet Autoencoder based Deep Neural Network", IEEE Access, vol.7, pp.46278-46287, 2019.

[4] Javaria Amin, Muhammad Sharif, Nadia Gul, Mussarat Yasmin, and Shafqat Ali Shad, "Brain tumor classification based on DWT fusion of MRI sequences using convolutional neural network", Pattern Recognition Letters, vol. 129, pp.115-122, 2020.

[5] Yin, B., Wang, C., and Abza, F, "New brain tumor classification method based on an improved version of whale optimization algorithm," Biomedical Signal Processing and Control, vol.56, 2020.

[6] Siva Raja, P. M., \& rani, A. V, "Brain tumor classification using a hybrid deep autoencoder with Bayesian fuzzy clustering-based segmentation approach," Biocybernetics and Biomedical Engineering, 2020.

[7] B. H. Menzeet al., "The Multimodal Brain Tumor Image Segmentation Benchmark (BRATS)," IEEE Transactions on Medical Imaging, vol. 34, no. 10, pp. 1993-2024, 2015.

[8] Khan, M.A., Ashraf, I., Alhaisoni, M., Damaševičius, R., Scherer, R., Rehman, A. and Bukhari, S.A.C., "Multimodal brain tumor classification using deep learning and robust feature selection: A machine learning application for radiologists", Diagnostics, vol.10, no.8, p.565, 2020.

[9] S. Bauer, R. Wiest, L.-P. Nolte, and M. Reyes, "A survey of MRI-based medical image analysis for brain tumor studies," Physicsinmedicine and biology, vol. 58, no. 13, pp. R97-R129, 2013.

[10] T. S. Armstrong, Z. Cohen, J. Weinberg, and M. R. Gilbert, "Imaging Techniques in Neuro-Oncology," Seminars in Oncology Nursing, vol. 20, no. 4, pp. 231-239, November 2004.

[11] John, P.,"Braintumor classification using wavelet and texture based neural network" International Journal of Scientific \& Engineering Research, vol.3, no.10, pp.1-7, 2012.

[12] Anitha V, and Murugavalli, S.,"Brain tumour classification using two-tier classifier with adaptive segmentation technique”, IET computer vision, vol. 10, no.1, pp.9-17, 2016

[13] Y. Li, F. Jia, and J. Qin, "Brain tumor segmentation from multimodal magnetic resonance images via sparse representation," Artificial Intelligence Med., vol. 73, pp. 1-13, 2016.

[14] X. Zhao, Y. Wu, G. Song, Z. Li, Y. Zhang, and Y. Fan, "A deep learning model integrating FCNNs and CRFs for brain tumor segmentation," Medical Image Analysis, vol. 43, pp. 98-111, 2018.

[15] Zhao, W., Zhang, Z. and Wang, L., "Manta ray foraging optimization: An effective bio-inspired optimizer for engineering applications," Engineering Applications of Artificial Intelligence, vol.87, pp.103300, 2020. 